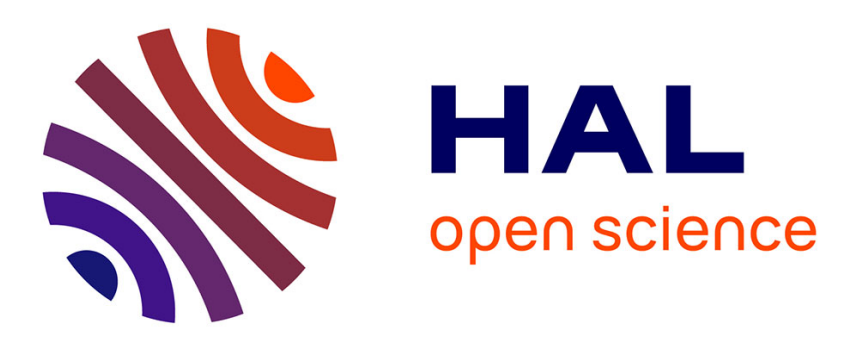

\title{
Breathing Dynamics for Systems of Interacting Particles in the Microcanonical and Canonical Descriptions
} Alain Olivetti, Julien Barré, B. Marcos, Freddy Bouchet, Robin Kaiser

\section{To cite this version:}

Alain Olivetti, Julien Barré, B. Marcos, Freddy Bouchet, Robin Kaiser. Breathing Dynamics for Systems of Interacting Particles in the Microcanonical and Canonical Descriptions. Transport Theory and Statistical Physics, 2010, 39, pp.524. hal-00822018

\section{HAL Id: hal-00822018 https://hal.science/hal-00822018}

Submitted on 13 May 2013

HAL is a multi-disciplinary open access archive for the deposit and dissemination of scientific research documents, whether they are published or not. The documents may come from teaching and research institutions in France or abroad, or from public or private research centers.
L'archive ouverte pluridisciplinaire HAL, est destinée au dépôt et à la diffusion de documents scientifiques de niveau recherche, publiés ou non, émanant des établissements d'enseignement et de recherche français ou étrangers, des laboratoires publics ou privés. 


\title{
BREATHING DYNAMICS FOR SYSTEMS OF INTERACTING PARTICLES IN THE MICROCANONICAL AND CANONICAL DESCRIPTIONS
}

\author{
A. OLIVETTI, J. BARRÉ, B. MARCOS, F. BOUCHET, AND R. KAISER
}

\begin{abstract}
By means of a dynamical ansatz, we study the breathing dynamics in systems of trapped interacting particles in a unified context, including a wide range of power law interactions and interaction strengths, at linear and non linear levels. We present detailed numerical tests of the general theory, and, motivated by Magneto-Optical Traps modeling, we extend it to the case of space dependent friction and diffusion.
\end{abstract}

\section{INTRODUCTION}

Low-lying oscillatory modes are a natural object of study for systems of trapped interacting particles; they are an important tool to understand and characterize the collective effects. As such, they have been studied in many different areas of physics: confined plasmas, trapped cold atoms, Bose-Einstein condensates, colloidal particles, trapped ions, astrophysical systems, the latter ones being self confined by the interactions. These systems feature a wide variety of interactions: weak or strong, long or short range.

We will be concerned in this paper with the simplest non trivial oscillatory mode for systems of trapped interacting particles in $d$ dimensions: the breathing mode [1]. It has been tackled in the literature using many different techniques, which we try to partially summarize here:

- a scaling ansatz to approximate the non linear dynamics of the BoltzmannVlasov equation for a classical gas is used in [2]; this idea was introduced earlier in the context of the Gross-Pitaevskii equation [3, 4].

- In the confined plasma context, the problem is often studied through hydrodynamical equations, in the so-called "cold fluid approximation" [5], where the dispersion relation for fluid modes in a cold spheroidal plasma is derived.

- Following an idea of [6], Ref. [7] gives an approximate solution to the breathing mode of a $d=1$ confined plasma beyond the cold fluid approximation, using an ad hoc closure of the hydrodynamical equations.

- Linearization of the Newtonian equations of motion around an equilibrium configuration and direct diagonalization have been used to study trapped ions or colloids interacting via a Coulomb or Yukawa potential in $d=1$ $[8,9], d=2[10,11]$ and $d=3[12]$. It has been generalized recently in [13] to a whole class of potentials. However, this technique is a priori restricted to the zero temperature limit and small perturbations.

Date: January 12, 2011. 
- Monopole modes of dusty plasmas interacting with a Yukawa potential are investigated in $[14,15]$, using a kind of mean-field approximation.

- Breathing oscillations with attractive interactions have been studied in an astrophysical context using the Virial theorem [16].

Each method applies to a specific situation: Newton equations are adapted to a crystallized state with negligible thermal fluctuations, linearization assumes a small amplitude of oscillations, the Vlasov equation is limited to weak correlations. Yet in all cases a similar equation for the breathing mode is obtained. In [1], we introduced a theory based on an extension of the scaling ansatz technique, which classifies many of the above examples in a common framework. In compensation this theory is mainly (but not entirely) limited to power law interactions, and does not give access to more complicated modes, beyond the monopole one. Figure 1 summarizes the different regimes we will study in this paper, assuming a binary isotropic power-law interparticle force $F(r) \sim 1 / r^{k}$ in $d$ space dimensions. On the horizontal axis is the interaction range, which we will call long-range if $k / d \leq 1$ and short range otherwise. The case $k / d \leq 1$ corresponds to non integrable forces at large distances [17]. The vertical axis represents the interaction strength with respect to the thermal energy. The third axis represents the friction, normalized by the trap frequency.

In this paper our goals are: $i$ ) to present extensive numerical tests of the theory developed in [1], investigating in particular its limits when the friction is increased; ii) motivated by magneto-optical traps modeling [18], to extend it to the case of space dependent friction and diffusion; iii) to test this extension of the theory using direct molecular dynamics simulations.

In section 2, we first review in details the theory already presented in [1], in a frictionless context, and provide comparisons with direct numerical simulations. In section 3, we introduce some friction and diffusion, and show that the equation for the breathing dynamics obtained with the ansatz method is exactly valid in the zero temperature limit, for all friction strength, for repulsive interactions. We then extensively test this equation against direct molecular dynamics simulations, to investigate its domain of validity. In section 4 , we extend the equation for the breathing dynamics to space dependent friction and diffusion, and test our results.

\section{BREATHING OSCILLATIONS WITHOUT FRICTION AND DIFFUSION}

2.1. Vlasov equation. We consider a system of particles confined by an harmonic spherical trapping force $\mathbf{F}_{\text {trap }}(\mathbf{r})=-\omega_{0}^{2} \mathbf{r}$, interacting with binary long range interaction forces $\mathbf{F}_{b i n}$. Let us first assume that correlations between particles are weak; we can then use the Vlasov equation to model the system in the continuum limit. In this section, we assume that the system is Hamiltonian.

Using the one-particle distribution $f(\mathbf{r}, \mathbf{v}, t)$, the Vlasov equation reads:

$$
\frac{\partial f}{\partial t}+\nabla_{\mathbf{r}} \cdot(\mathbf{v} f)+\mathbf{F}_{\text {trap }} \cdot \nabla_{\mathbf{v}} f+\mathbf{F}_{\text {int }}[f] \cdot \nabla_{\mathbf{v}} f=0
$$

where $\mathbf{F}_{\text {int }}[f]$ is the interaction term given by:

$$
\mathbf{F}_{i n t}[f](\mathbf{r})=\int \mathbf{F}_{b i n}(\mathbf{r}, \tilde{\mathbf{r}}) f(\tilde{\mathbf{r}}, \mathbf{v}, t) d \tilde{\mathbf{r}} d \mathbf{v} .
$$

We consider $f_{0}$, a stationary state solution of Eq. (1). If $\mathbf{F}_{b i n}=-\nabla_{\mathbf{r}} V_{b i n}$ is a potential force, then $\mathbf{F}_{\text {int }}[f]=-\nabla_{\mathbf{r}} V_{\text {int }}[f]$ is also potential, and a natural choice 


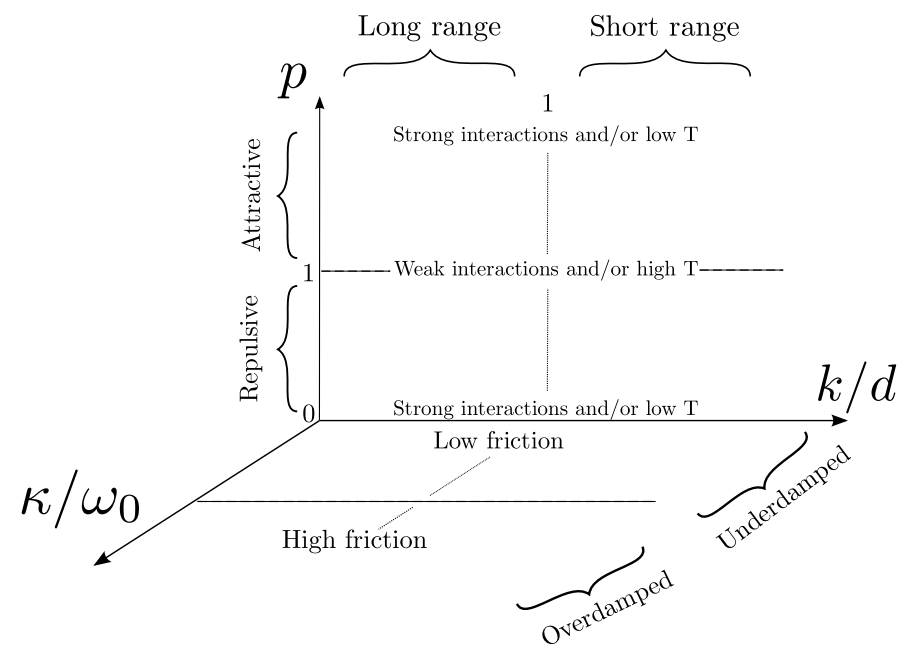

Figure 1. (color online). Diagram of the different regimes for the breathing dynamics. On the horizontal axis, the interaction range, measured by $k / d$. The interaction strength is changing along the vertical axis. The third axis represents the friction normalized by the trap frequency $\kappa / \omega_{0}$.

for $f_{0}$ is the statistical equilibrium, parametrized by the inverse temperature $\beta$. This statistical equilibrium is implicitly defined by the equation

$$
f_{0}(\mathbf{r}, \mathbf{v}) \propto e^{-\beta \mathbf{v}^{2} / 2} e^{-\beta V_{i n t}\left[f_{0}\right](\mathbf{r})} e^{-\beta \omega_{0}^{2} \mathbf{r}^{2} / 2}
$$

Such an equilibrium does not always exist when the interaction is attractive. We will consider this statistical equilibrium for $f_{0}$ in this subsection, unless explicitly stated. We also assume that $f_{0}$ is isotropic in positions.

We now drastically simplify the dynamics by using a scaling ansatz $[2,3,4]$ :

$$
f(\mathbf{r}, \mathbf{v}, t)=f_{0}(\varphi(\mathbf{r}, \mathbf{v})),
$$

with

$$
\varphi(\mathbf{r}, \mathbf{v})=(\mathbf{R}=\mathbf{r} / \lambda, \mathbf{V}=\lambda \mathbf{v}-\dot{\lambda} \mathbf{r}) .
$$

With this hypothesis all the time dependence in the dynamics is now included in the positive parameter $\lambda$. This ansatz is tailored to capture the radial dynamics; thus, we will not be able to look at higher order modes of the system, like quadrupole modes.

We justify the ansatz as follows. We assume that the spatial dynamics can be described with the simple scaling ansatz

$$
\mathbf{R}=\frac{\mathbf{r}}{\lambda(t)}
$$

In the Hamiltonian description, we require the conservation of the phase space volume, i.e., $d \mathbf{r} d \mathbf{v}=d \mathbf{R} d \mathbf{V}$. This implies that

$$
\mathbf{V}=\lambda(t) \mathbf{v}+\mathbf{h}(\mathbf{r}, t),
$$


where $\mathbf{h}$ is an arbitrary function of $\mathbf{r}$ and $t$. Integrating the Vlasov equation over the velocity variable, we obtain the continuity equation

$$
\frac{\partial \rho(\mathbf{r}, t)}{\partial t}+\frac{\partial(\mathbf{u}(\mathbf{r}, t) \rho(\mathbf{r}, t))}{\partial \mathbf{r}}=0,
$$

where the space density $\rho$ and the velocity $\mathbf{u}$ are defined by

$$
\left\{\begin{array}{l}
\rho(\mathbf{r}, t)=\int f(\mathbf{r}, \mathbf{v}, t) d \mathbf{v} \\
\rho(\mathbf{r}, t) \mathbf{u}(\mathbf{r}, t)=\int \mathbf{v} f(\mathbf{r}, \mathbf{v}, t) d \mathbf{v} .
\end{array}\right.
$$

Calling $\rho_{0}(\mathbf{r})$ the stationary solution for the density $\rho(\mathbf{r}, t)$, and injecting the scaling ansatz inside Eq. (8), we have:

(10) $\frac{\partial}{\partial t}\left[\frac{1}{\lambda^{d}} \rho_{0}\left(\frac{\mathbf{r}}{\lambda}\right)\right]+\frac{1}{\lambda^{d+1}} \frac{\partial}{\partial \mathbf{r}}\left[\rho_{0}\left(\frac{\mathbf{r}}{\lambda}\right) \mathbf{u}\left(\frac{\mathbf{r}}{\lambda}, t\right)\right]-\frac{1}{\lambda^{d+1}} \frac{\partial}{\partial \mathbf{r}}\left[\mathbf{h}(\mathbf{r}, t) \rho_{0}\left(\frac{\mathbf{r}}{\lambda}\right)\right]=0$.

Assuming that $f_{0}$ is even with respect to its velocity variables (which is the case for (3)), the second term of Eq. (10) vanishes. Looking for a condition over $\mathbf{h}$ which cancels separately the terms containing $\rho_{0}$ and $\partial_{\mathbf{r}} \rho_{0}$, we obtain finally

$$
\mathbf{h}(\mathbf{r}, t)=-\dot{\lambda}(t) \mathbf{r} .
$$

Combining Eq. (6), (7) and (11) leads to the scaling ansatz (4).

After having given this justification of the scaling ansatz (4), let us now insert it into Eq. (1). This leads to:

$$
\sum_{i=1}^{d}\left\{\frac{V_{i}}{\lambda^{2}} \frac{\partial f_{0}}{\partial R_{i}}-R_{i} \lambda \frac{\partial f_{0}}{\partial V_{i}}\left(\ddot{\lambda}+\omega_{0}^{2} \lambda\right)+\lambda \mathbf{F}_{\text {int }}\left[f_{0} \circ \varphi\right](\mathbf{r}) . \nabla_{\mathbf{V}} f_{0}\right\}=0 .
$$

We now assume that the binary interaction is homogeneous with degree $-k$ (we use here and in the following the word "homogeneous" in its mathematical sense):

$$
\mathbf{F}_{b i n}(\lambda \mathbf{r}, \lambda \tilde{\mathbf{r}})=\frac{1}{\lambda^{k}} \mathbf{F}_{b i n}(\mathbf{r}, \tilde{\mathbf{r}}) .
$$

The previous relation eliminates the function $\varphi$ in the interaction term:

$$
\begin{aligned}
\mathbf{F}_{i n t}\left[f_{0} \circ \varphi\right](\mathbf{r}) & =\int_{\mathbf{F}_{b i n}(\mathbf{r}, \lambda \tilde{\mathbf{r}}) f_{0}(\tilde{\mathbf{r}}, \mathbf{v}) d \tilde{\mathbf{r}} d \mathbf{v}} \\
& =\frac{1}{\lambda^{k}} \int \mathbf{F}_{b i n}(\mathbf{R}, \tilde{\mathbf{r}}) f_{0}(\tilde{\mathbf{r}}, \mathbf{v}) d \tilde{\mathbf{r}} d \mathbf{v} \\
& =\frac{1}{\lambda^{k}} \mathbf{F}_{i n t}\left[f_{0}\right](\mathbf{R}) .
\end{aligned}
$$

Finally, using Eq. (14) and the fact that $f_{0}$ is a stationary solution of Eq. (1), one can substitute the interaction term by a linear combination of $f_{0}$ and its derivatives. Equation (12) becomes

$$
\sum_{i=1}^{d} V_{i} \frac{\partial f_{0}}{\partial R_{i}}\left(\frac{1}{\lambda^{2}}-\lambda^{1-k}\right)-R_{i} \frac{\partial f_{0}}{\partial V_{i}}\left[\lambda\left(\ddot{\lambda}+\omega_{0}^{2} \lambda\right)-\lambda^{1-k} \omega_{0}^{2}\right]=0 .
$$

Multiplying it by $R_{j} V_{j} / N$, and integrating over $d \mathbf{R} d \mathbf{V}$, leads to a constraint on the parameter $\lambda$ :

$$
\ddot{\lambda}+\left(\lambda-\frac{1}{\lambda^{k}}\right) \omega_{0}^{2}-\left(\frac{1}{\lambda^{3}}-\frac{1}{\lambda^{k}}\right) \frac{\left\langle V_{j}^{2}\right\rangle_{f_{0}}}{\left\langle R_{j}^{2}\right\rangle_{f_{0}}}=0
$$


where $j$ is a coordinate label, and we have set

$$
\langle\chi\rangle_{f}=\frac{1}{N} \int \chi(\mathbf{r}, \mathbf{v}) f(\mathbf{r}, \mathbf{v}, t) d \mathbf{r} d \mathbf{v} .
$$

In the dynamical equation for $\lambda(16)$, all parameters are computed as averages over the stationary distribution $f_{0}$. Since $f_{0}$ is isotropic in positions and velocities, $\left\langle V_{j}^{2}\right\rangle_{f_{0}}$ and $\left\langle R_{j}^{2}\right\rangle_{f_{0}}$ do not depend on $j$ : Eq. (16) is the same for the $d$ coordinates.

Taking higher order moments of Eq. (15) would produce different equations for $\lambda$, implying that the only solution is the trivial one $\lambda=1$. This is a consequence of the fact that the ansatz does not contain any non trivial exact solution of the Vlasov equation. One may hope however that Eq. (16) satisfactorily describes the breathing dynamics over short times: this will be tested in numerical simulations.

A dimensionless parameter appears naturally in Eq. (16), which we define as

$$
p=\frac{\left\langle V_{j}^{2}\right\rangle_{f_{0}}}{\omega_{0}^{2}\left\langle R_{j}^{2}\right\rangle_{f_{0}}} .
$$

When $f_{0}$ is given by $(3)$, it can be interpreted as the ratio between the thermal energy $k_{B} T$ and the typical potential energy due to the trap $E_{\text {trap }} \sim \omega_{0}^{2}\left\langle R^{2}\right\rangle_{f_{0}}$ :

$$
p \sim \frac{k_{B} T}{E_{\text {trap }}} .
$$

Using (3) for a system with and without interactions $\left(V_{\text {int }}=0\right)$, at the same temperature, we have

$$
\left\langle V_{j}^{2}\right\rangle_{f_{0}, V_{i n t}=0}=\left\langle V_{j}^{2}\right\rangle_{f_{0}}=\omega_{0}^{2}\left\langle R_{j}^{2}\right\rangle_{f_{0}, V_{i n t}=0}
$$

which implies

$$
p=\frac{\left\langle R_{j}^{2}\right\rangle_{f_{0}, V_{i n t}=0}}{\left\langle R_{j}^{2}\right\rangle_{f_{0}}}
$$

We can summarize this:

- $0<p<1$ corresponds to repulsive interactions, with $p \ll 1$ the strong interaction regime.

- If $p \sim 1$, the system is weakly interacting.

- For $p>1$, the interaction is attractive, and $p \gg 1$ corresponds to the strong interaction regime.

We now write (16) as

$$
\ddot{\lambda}+\left(\lambda-\frac{p}{\lambda^{3}}+\frac{p-1}{\lambda^{k}}\right) \omega_{0}^{2}=0 .
$$

2.2. Extension of the ansatz. In the previous subsection, we started from the Vlasov equation. In order to take better into account correlations between particles (and thus describe also short range interacting systems) we start now from the first equation of the Bogolyubov-Born-Green-Kirkwood-Yvon (BBGKY) hierarchy:

$$
\frac{\partial f}{\partial t}+\nabla_{\mathbf{r}} \cdot(\mathbf{v} f)+\mathbf{F}_{\text {trap }} \cdot \boldsymbol{\nabla}_{\mathbf{v}} f+C[g]=0,
$$

where $C[g]$ is the interaction term which is given now by:

$$
C[g]\left(\mathbf{r}_{1}, \mathbf{v}_{1}, t\right)=\int \mathbf{F}_{b i n}\left(\mathbf{r}_{1}, \mathbf{r}\right) . \nabla_{\mathbf{v}_{1}} g\left(\mathbf{r}_{1}, \mathbf{v}_{1}, \mathbf{r}, \mathbf{v}, t\right) d \mathbf{r} d \mathbf{v}
$$


and $g\left(\mathbf{r}_{1}, \mathbf{v}_{1}, \mathbf{r}, \mathbf{v}, t\right)$ the two-particles distribution. We stress that Eq. (21), in contrast with the Vlasov equation, can also describe the dynamics of strongly correlated systems and also short range interacting systems. Note that Eq. (21) is strictly equivalent to the Hamiltonian equation because we have not yet done any hypothesis on the unknown function $g$. We assume as above the existence of a stationary state $f_{0}$ and $g_{0}$, for instance the statistical equilibrium, and perform the closure using the previous scaling ansatz (4), extended to the two-particles function $g$, as done in [1]:

$$
\left\{\begin{array}{l}
f\left(\mathbf{r}_{1}, \mathbf{v}_{1}, t\right)=f_{0}\left(\varphi\left(\mathbf{r}_{1}, \mathbf{v}_{1}\right)\right) \\
g\left(\mathbf{r}_{1}, \mathbf{v}_{1}, \mathbf{r}_{2}, \mathbf{v}_{2}, t\right)=g_{0}\left(\psi\left(\mathbf{r}_{1}, \mathbf{v}_{1}, \mathbf{r}_{2}, \mathbf{v}_{2}\right)\right)
\end{array}\right.
$$

with

$$
\left\{\begin{array}{l}
\varphi\left(\mathbf{r}_{1}, \mathbf{v}_{1}\right)=\left(\mathbf{R}_{1}=\mathbf{r}_{1} / \lambda, \mathbf{v}_{1}=\lambda \mathbf{v}_{1}-\dot{\lambda} \mathbf{r}_{1}\right) \\
\psi\left(\mathbf{r}_{1}, \mathbf{v}_{1}, \mathbf{r}_{2}, \mathbf{v}_{2}\right)=\left(\varphi\left(\mathbf{r}_{1}, \mathbf{v}_{1}\right), \varphi\left(\mathbf{r}_{2}, \mathbf{v}_{2}\right)\right) .
\end{array}\right.
$$

Once again, the positive parameter $\lambda$ contains all the time dependence of the dynamics. The computations are similar to those of subsection 2.1. Introducing the ansatz in Eq. (21) yields:

$$
\sum_{i=1}^{d}\left\{\frac{V_{i}}{\lambda^{2}} \frac{\partial f_{0}}{\partial R_{i}}-R_{i} \lambda \frac{\partial f_{0}}{\partial V_{i}}\left(\ddot{\lambda}+\omega_{0}^{2} \lambda\right)+C\left[g_{0} \circ \psi\right]\left(\mathbf{r}_{1}, \mathbf{v}_{1}, t\right)\right\}=0 .
$$

The homogeneity of the binary forces (see Eq.(13)) allows us to simplify the interaction term:

$$
\begin{aligned}
C\left[g_{0} \circ \psi\right](\mathbf{r}, \mathbf{v}) & =\int \mathbf{F}_{b i n}(\lambda \mathbf{R}, \lambda \mathbf{S}) . \nabla_{\mathbf{v}}\left[g_{0}(\mathbf{R}, \mathbf{V}, \mathbf{S}, \mathbf{W})\right] d \mathbf{S} d \mathbf{W} \\
& =\lambda \int \mathbf{F}_{b i n}(\lambda \mathbf{R}, \lambda \mathbf{S}) . \nabla_{\mathbf{v}} g_{0}(\mathbf{R}, \mathbf{V}, \mathbf{S}, \mathbf{W}) d \mathbf{S} d \mathbf{W} \\
& =\lambda^{1-k} C\left[g_{0}\right](\mathbf{R}, \mathbf{V}) .
\end{aligned}
$$

We now multiply by $R_{j} V_{j} / N$, and integrate over $d \mathbf{R} d \mathbf{V}$. Introducing, as before, the dimensionless parameter $p$ defined in Eq. (18), we obtain the equation:

$$
\ddot{\lambda}+\left(\lambda-\frac{p}{\lambda^{3}}+\frac{p-1}{\lambda^{k}}\right) \omega_{0}^{2}=0,
$$

which is exactly the same as Eq. (20).

2.3. Analysis of the breathing dynamics. We can rewrite Eq. (20) as an equation of an anharmonic oscillator in the external potential $\phi$ :

$$
\ddot{\lambda}+\phi^{\prime}(\lambda)=0
$$

with

$$
\phi(\lambda)= \begin{cases}\omega_{0}^{2}\left(\frac{1}{2} \lambda^{2}+\frac{1}{2} \frac{p}{\lambda^{2}}+\frac{p-1}{1-k} \lambda^{1-k}\right), & \text { if } k \neq 1, \\ \omega_{0}^{2}\left(\frac{1}{2} \lambda^{2}+\frac{1}{2} \frac{p}{\lambda^{2}}+(p-1) \log \lambda\right), & \text { if } k=1 .\end{cases}
$$

The first term in Eq. (29) is the quadratic confining potential, the second one corresponds to a kinetic pressure term (which does no depend on the dimension $d$ considered) and the last one is introduced by the two-body interaction.

The shape of the potential determines the form of the oscillations. For repulsive interactions $(p<1)$, the potential is convex for all $k$. It diverges as $\phi \sim \lambda^{-2}$ as $\lambda \rightarrow 0$ and behaves as $\phi \sim \lambda^{2}$ as $\lambda \rightarrow \infty$, which ensures that the system oscillates around its unique minimum $\lambda=1$. For attractive $(p>1)$ interactions and 
$0 \leqslant k \leqslant 3$, the potential presents the same properties. For attractive interactions and $k>3$, the potential diverges to $-\infty$ as $\lambda^{1-k}$ for small $\lambda$. If $p<1-4 /(3-k)$, $\lambda=1$ is a metastable stationary state, and there exists an unstable stationary state for $\lambda^{*}<1$, see Fig. 2. For $p>1-4 /(3-k), \lambda=1$ is an unstable stationary state and there is a metastable state for $\lambda>1$.

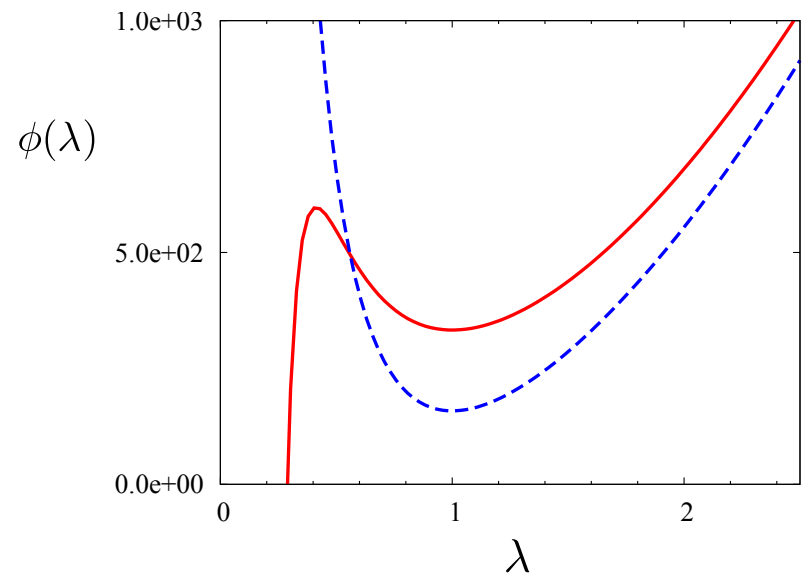

Figure 2. (color online). Shape of the potential for two different cases: repulsive (dotted line) and attractive with $k>3$ (solid line).

From Eq. (16), we obtain the general expression of the linearized breathing oscillation frequency as a function of the interaction range $k$ and the interaction strength $p$ :

$$
\omega(k, p)=\omega_{0}[(3-k)(p-1)+4]^{1 / 2}
$$

This expression recovers the well known limits $\omega=2 \omega_{0}$ for a non interacting gas $(p=1)$ and $\omega=\sqrt{3} \omega_{0}$ for a strongly interacting Coulomb plasma $(p=0, k=2)$ [20]. It provides a generalization to the whole $(k, p)$ plane shown in Fig. 1 and is independent of the dimension. We note that for $k>3$ and repulsive interactions $(p<1)$, the breathing frequency is an increasing function of the interaction strength (decreasing function of $p$ ); this corresponds in 3 dimensions to short range interactions $(k>d)$. On the other hand, for $k<3$ it is a decreasing function of the interaction strength (increasing function of $p$ ); this corresponds in 3 dimensions to long range interactions $(k<d)$. See Fig. 3 for details.

2.4. Comparison with the literature. We can now compare Eqs. (16) and (27) to the results found in the literature for various specific situations. Oscillations of crystallized systems $[8,9,11,13]$ correspond to negligible pressure effects, i.e. $p=0$ and the $\lambda^{-3}$ term of is absent. In [7], the authors consider a $d=1$ plasma $(k=0)$ with $p$ not too small, and introduce a pressure yielding the $\lambda^{-3}$ term, which leads to the exact equivalent of Eq. (16).

In [2] is considered a classical gas with "mean field" interactions, given by a Dirac $\delta$ potential. This corresponds to a homogeneity degree for the force $-k=-d-1$. Equation (16) contains this case, and this emphasizes that the present theory is not only valid for power-law forces. 


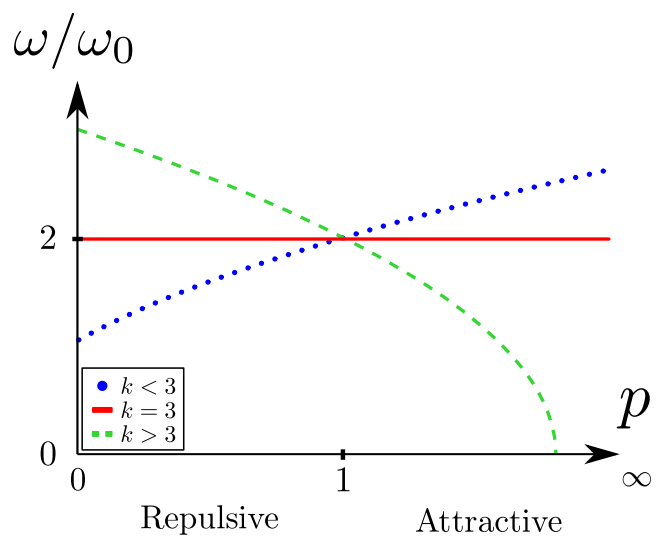

Figure 3. (color online). Frequency of the linearized breathing mode as a function of the interaction strength $p$, for different values of interaction range $k$.

The Yukawa potential

$$
V(\mathbf{r}) \propto \frac{\exp \left(-|\mathbf{r}| / L_{s}\right)}{|\mathbf{r}|}
$$

is not homogeneous, so that our method does not work. However, both in the Coulombian limit, where the shielding length $L_{s}$ is much larger than the system size, and the opposite one, where $L_{s}$ is much smaller than the system size, the Yukawa potential may be approximated by a homogeneous potential, respectively a Coulomb and a Dirac $\delta$ potential. Equation (16) then reproduces the results of $[14,15]$, obtained by other means.

2.5. Comparison with numerical simulations. In order to test the domain of validity of Eq. (27), we have performed several numerical simulations. We have considered different force index $k$, parameter $p$ and amplitude of initial perturbation, in one, two and three dimensions.

We simulate the system using a molecular dynamics approach with $N=4000$ particles unless otherwise stated. The integrator scheme is a Verlet-leapfrog algorithm [21] in the micro-canonical ensemble. The forces are exactly computed at each time-step.

The computer simulations are performed as follows: we first equilibrate the system in a stationary state using a Langevin thermostat (see section 3.3). Then, at $t=0$, we introduce a perturbation by rescaling the positions and velocities according to Eqs. (4) and (5), and we let the system evolve. A similar simulation of a $d=1$ Coulomb system has been performed in [7], corresponding to cases with $k / d=0$ and $p<1$. In the following we present the results of the simulations and a comparison with the theory developed here. We have studied most of the possible combinations between (i) repulsive or attractive interactions (ii) short or long range interactions (iii) weak or strong interactions (iv) small or large amplitudes oscillations. Figure 4 shows some of our numerical tests which we describe in the following: 
BREATHING DYNAMICS FOR SYSTEMS OF INTERACTING PARTICLES IN THE MICROCANONICAL AND CANONICAL DES

- We have extensively studied the case in which the interaction is repulsive. The results are the following, classified according to the interaction strength: for strong interactions $(p \ll 1)$ the ansatz describes very well both the amplitude and frequency of oscillation, for all the cases considered, and that for many oscillations. This is because the ansatz is exact in the limit $p \rightarrow 0$, as we will show in subsection 3.1. We include two examples (Figs. 4(a) with a very small $p$ and 4(b) for $p=0.166$ ), respectively with $d=3$ and $d=2$, both of them for small amplitude oscillations and short-range interaction. Long-range and/or large amplitude oscillations leads to the same conclusions.

When $p$ is of order 1 , the ansatz gives a less accurate description of the simulations. It is in general able to predict the frequency of several oscillations, but gives less good results for the amplitudes. The ansatz (4) predicts a self-similar evolution of the density:

$$
\rho(\mathbf{r}, t)=\lambda^{-d} \rho_{0}\left(\frac{\mathbf{r}}{\lambda}\right)
$$

It is therefore not able to describe, e.g., the evolution of the density if particles which are in a given shell of the initial (spherical) distribution are transported during the evolution to another shell (which is called "shell crossing" in fluid mechanics, see e.g. [22]). For long range interactions, such phenomena occurs during the so called "violent relaxation", which is a well known process of relaxation to a quasi-stationary state [23]. Figures. 4(c) and 4(d) illustrate this situation for long-range interaction and different initial perturbations. The ansatz predicts no decay of the oscillation amplitude, whereas the simulations show such decay: we may then attribute this to the "violent relaxation" phenomenon. In the case of short range interactions, a similar approximate description by the ansatz of the simulations is observed. In this case, a mechanism candidate for the loss of coherence in the oscillations (and hence their decay) is two-body collisions, which is an efficient relaxation process in such short range interacting systems.

- For numerical reasons, we only simulated in the attractive case long-range interacting systems. We show such a numerical experiment in Fig. 4(e) and 4 (f), for $k=0$ and $p=2.2$ ( $p=70$ for inset) in $d=3$. In these cases the "violent relaxation" is always important, which explains the decay in the oscillation amplitude observed in the simulations and not predicted by the ansatz. Finally remark that a large amplitude oscillation leads to a worse prediction than in the repulsive case.

On Figs. 4(c)-4(f), we see that the asymptotic value of $\left\langle R^{2}\right\rangle_{f} /\left\langle R^{2}\right\rangle_{f_{0}}$ seems to be different from 1 , indicating a relaxation to a stationary state different from the initial one. This was to be expected, as the initial perturbation changes the energy of the system with respect to the reference state $f_{0}$, and there is no dissipation. Since the ansatz is built on the reference stationary state $f_{0}$, one cannot hope that it will be relevant to describe the asymptotic stationary state.

On a 3D system with Coulomb interactions, we have checked that our numerical results do not change significantly performing simulations with $N=1000$ and $N=10000$ particles. After 10 oscillations, we observe a difference around $1 \%$ in the amplitude and no sizable difference for the frequencies. 


\section{BREATHING OSCILLATIONS WITH FRICTION AND DIFFUSION}

In many cases, it is reasonable to assume that particles, rather than following a Hamiltonian evolution, are subjected to small random uncorrelated forces, and a friction: this situation is conveniently modeled by adding to the Vlasov equation a Fokker-Planck operator with a positive constant friction $\kappa$ and diffusion $D$, which amounts to define a temperature.

3.1. Small $T$ limit for repulsive interactions. In this subsection we consider the zero temperature limit of the model for a repulsive interaction, i.e., the limit in which $p \rightarrow 0^{1}$.This is also the limit where the random force is negligible, so that the dynamics is described by Newton equations.

In this case, the reference stationary state will be a stationary configuration of the particles. Let us consider such a stationary configuration $\left\{\mathbf{r}_{i}^{0}\right\}_{i=1}^{N}$ for the $N$ particles. The force $\mathbf{F}_{i}^{0}$ on each particle therefore vanishes:

$$
\mathbf{F}_{i}^{0}=\mathbf{F}_{\text {trap }}\left(\mathbf{r}_{i}^{0}\right)+\sum_{j \neq i} \mathbf{F}_{b i n}\left(\mathbf{r}_{j}^{0}-\mathbf{r}_{i}^{0}\right)=\mathbf{0} .
$$

We consider now a breathing dynamics $\mathbf{r}_{i}(t)=\lambda(t) \mathbf{r}_{i}^{0}$. Newton equations read

$$
\begin{aligned}
\ddot{\lambda} \mathbf{r}_{i}^{0} & =\mathbf{F}_{\text {trap }}\left(\lambda(t) \mathbf{r}_{i}^{0}\right)-\kappa \dot{\lambda} \mathbf{r}_{i}^{0}+\sum_{j \neq i} \mathbf{F}_{b i n}\left(\lambda \mathbf{r}_{j}^{0}-\lambda \mathbf{r}_{i}^{0}\right) \\
& =-\omega_{0}^{2} \lambda \mathbf{r}_{i}^{0}-\kappa \dot{\lambda} \mathbf{r}_{i}^{0}+\lambda^{-k} \sum_{j \neq i} \mathbf{F}_{b i n}\left(\mathbf{r}_{j}^{0}-\mathbf{r}_{i}^{0}\right) \\
& =-\omega_{0}^{2} \lambda \mathbf{r}_{i}^{0}-\kappa \dot{\lambda} \mathbf{r}_{i}^{0}+\omega_{0}^{2} \lambda^{-k} \mathbf{r}_{i}^{0}
\end{aligned}
$$

From the first equation to the second one, we have used the homogeneity of the binary force; from the second one to the third, we have used Eq. (33) to express the interaction term as a function of the trap force. This yields an equation for $\lambda$

$$
\ddot{\lambda}=-\kappa \dot{\lambda}+\omega_{0}^{2}\left(\lambda^{-k}-\lambda\right),
$$

Putting $\kappa=0$ in Eq. (37) and $p=0$ in Eq. (20), one finds the same equation. This calculation is very similar to the one done in [13]; in this reference, the authors linearize the particles motion, thus limiting themselves to small deviations from the reference state. We have shown here that this restriction is unnecessary.

3.2. Vlasov-Fokker-Planck equation. When the random force is not negligible, our new starting point is now the Vlasov-Fokker-Planck equation:

$$
\frac{\partial f}{\partial t}+\boldsymbol{\nabla}_{\mathbf{r}} \cdot(\mathbf{v} f)+\mathbf{F}_{\text {trap }} \cdot \boldsymbol{\nabla}_{\mathbf{v}} f+\mathbf{F}_{\text {int }}[f] \cdot \boldsymbol{\nabla}_{\mathbf{v}} f=D \Delta_{\mathbf{v}} f+\kappa \boldsymbol{\nabla}_{\mathbf{v}} \cdot(\mathbf{v} f) .
$$

Once again, we assume the existence of a stationary state $f_{0}$ solution of Eq. (38). If the binary interaction is potential and repulsive, there is indeed a unique stationary state of (38), given by the canonical statistical equilibrium (3), see for instance [24], appendix E.

As done in section 2.2, the Vlasov part on the left hand side of Eq. (38) may be replaced by the first equation of the BBGKY hierarchy. We will not repeat these computations here.

\footnotetext{
${ }^{1}$ This limit for an attractive interaction, $p \rightarrow \infty$, is trivial: all the particles collapse on a point in the center of the trap.
} 
Inserting into (38) the same scaling ansatz as in section 2.1, and performing the same computation, one obtains the following equation:

$$
\begin{aligned}
& \sum_{i=1}^{d}\left\{V_{i} \frac{\partial f_{0}}{\partial R_{i}}\left(\frac{1}{\lambda^{2}}-\lambda^{1-k}\right)+D \frac{\partial^{2} f_{0}}{\partial V_{i}^{2}}\left(\lambda^{1-k}-\lambda^{2}\right)\right. \\
& \left.-R_{i} \frac{\partial f_{0}}{\partial V_{i}}\left[\lambda\left(\ddot{\lambda}+\omega_{0}^{2} \lambda\right)-\lambda^{1-k} \omega_{0}^{2}+\kappa \lambda \dot{\lambda}\right]+\kappa \frac{\partial\left(V_{i} f_{0}\right)}{\partial V_{i}}\left(\lambda^{1-k}-1\right)\right\}=0 .
\end{aligned}
$$

Taking the moment $R_{j} V_{j}$ of this equation yields the equation for $\lambda$ :

$$
\ddot{\lambda}+\kappa \dot{\lambda}+\left(\lambda-\frac{p}{\lambda^{3}}+\frac{p-1}{\lambda^{k}}\right) \omega_{0}^{2}=0,
$$

where we have used for $p$ the same definition as above (18). As in the case without friction, taking higher order moments of Eq. (39) yields different equations for $\lambda$, implying $\lambda=1$. However, at variance with the case without friction, already the second order moment $V_{j}^{2}$ implies that the only exact solution compatible with the ansatz is $\lambda=1$. We also note that Eq. (40) is compatible with Eqs. (20) and (37) respectively in the limits $\kappa \rightarrow 0$ and $p \rightarrow 0$. As a consequence, one may expect Eq. (40) to give useful information on the breathing dynamics at small friction or small $p$, but its precise domain of validity has to be investigated numerically.

3.3. Comparison with numerical simulations. We have performed the numerical simulations in the same setting as in section 2.5, adding a thermostat. We use a Langevin - Verlet numerical scheme in which the force during each time-step is assumed to vary linearly with time, as the one described in [21]. Our goal is to investigate in which regions of the $\left(p, k / d, \kappa / \omega_{0}\right)$ space the scaling ansatz can be useful, see Fig 1 . We have used $N=1000$ and $N=5000$ particles considering successively one, two or three space dimension, with $k=0$. In each case we have performed the simulations varying $p$ and $\kappa$ following a grid in the plane $\left(\kappa / \omega_{0}, p\right)$, restricting ourselves to repulsive interactions.

In section 2.5, we have seen that the constant energy simulations showed a very good agreement with the reduced dynamics as far as the oscillation frequency is concerned, and some disagreement concerning the oscillation amplitude. Since we would like to assert here the effect of friction and diffusion, we concentrate on the oscillation frequencies, and use the following criterion to estimate the agreement or disagreement between the simulation and the reduced dynamics Eq. (40):

- In the underdamped regime we consider the relative difference between the theoretical and numerical times for the $n^{t h}$ maximum of the oscillation: $\left|t_{\text {theo }}^{n}-t_{\text {num }}^{n}\right| / t_{\text {theo }}^{n}$. We choose $n$ to be the last maximum above the noise level.

- In the overdamped regime we consider the half-life time of the initial perturbation: $\left|t_{\text {theo }}^{1 / 2}-t_{\text {num }}^{1 / 2}\right| / t_{\text {theo }}^{1 / 2}$.

Fig. 5 confirms that for $k=0$ close to axes $p=0$ and/or $\kappa / \omega_{0}=0$ the scaling ansatz method leads to reasonable predictions. On the contrary when we approach an overdamped dynamics, the scaling ansatz rapidly fails. We expect a similar picture for different values of $k$. Fig. 6 represents some examples in the underdamped regime for different systems. Frequencies are quite precisely predicted by the ansatz, even if the amplitude's decay is not negligible. Note that contrary to simulations done in section 2.5, the initial state $f_{0}$ is also the final one, since the system has a unique stationary state. 


\section{SPACE DEPENDENT FRICTION}

We extend in this section the study of the breathing dynamics with the ansatz method to the case where the friction coefficient $\kappa$ as well as the diffusion constant $D$ are space-dependent. One physical motivation for this extension is the physics of Magneto-Optical Traps (MOTs). In an atomic cloud confined in a MOT, the interaction of atoms with lasers induces a friction and a diffusion; however, it is known that the intensity of these friction and diffusion depends on the atom position, and that it may have important dynamical consequences [18]. Although we will concentrate here on the effect of a space-dependent friction, a precise modeling of a MOT would clearly require to take into account other effects.

4.1. Dynamical equation. Our goal is to obtain an effective dynamical equation for the breathing dynamics similar to Eq (40), which would also be valid in both the small friction and strong repulsive interaction limits.

Eq. (40) was obtained taking the $R_{i} V_{i}$ moment of Eq. (39), which in turn came from the use of the dynamical ansatz. When the friction was homogeneous, it was then possible to check that Eq. (40) was compatible with the exact solution at $p=0$ (or $T=0$ ) Eq (37). When friction and diffusion are not homogeneous, taking the $R_{i} V_{i}$ moment after introducing the dynamical ansatz yields an effective equation analogous to Eq. (40) but we do not have any more an exact solution at $p=0$ to test its consistency. We will thus rely again on numerical simulations to investigate its domain of validity.

We first introduce the dynamical ansatz into the equation with inhomogeneous friction and diffusion.

We start from the Vlasov Fokker-Planck equation including the space dependence $\kappa(\mathbf{r})$ and $D(\mathbf{r})$ (once again it is straightforward to do the same thing considering the first equation of the BBGKY hierarchy):

$$
\frac{\partial f}{\partial t}+\nabla_{\mathbf{r}} \cdot(\mathbf{v} f)+\mathbf{F}_{\text {trap }} \cdot \nabla_{\mathbf{v}} f+\mathbf{F}_{i n t}[f] \cdot \nabla_{\mathbf{v}} f=D(\mathbf{r}) \Delta_{\mathbf{v}} f+\kappa(\mathbf{r}) \boldsymbol{\nabla}_{\mathbf{v}} \cdot(\mathbf{v} f),
$$

We assume again the existence of a stationary state $f_{0}$. When friction and diffusion are not homogeneous, we have no simple implicit equation for $f_{0}$ such as $(3)$.

Using the scaling ansatz method with stationary state $f_{0}$ leads to the equivalent equation of Eq. (39):

$$
\begin{array}{r}
\sum_{i=1}^{d}\left\{V_{i} \frac{\partial f_{0}}{\partial R_{i}}\left(\frac{1}{\lambda^{2}}-\lambda^{1-k}\right)+\frac{\partial^{2} f_{0}}{\partial V_{i}^{2}}\left[\lambda^{1-k} D(\mathbf{R})-\lambda^{2} D(\lambda \mathbf{R})\right]\right. \\
-R_{i} \frac{\partial f_{0}}{\partial V_{i}}\left[\lambda\left(\ddot{\lambda}+\omega_{0}^{2} \lambda\right)-\lambda^{1-k} \omega_{0}^{2}+\kappa(\lambda \mathbf{R}) \lambda \dot{\lambda}\right] \\
\left.+\frac{\partial\left(V_{i} f_{0}\right)}{\partial V_{i}}\left[\lambda^{1-k} \kappa(\mathbf{R})-\kappa(\lambda \mathbf{R})\right]\right\}=0
\end{array}
$$

We now multiply Eq. (42) by $R_{j} V_{j} / N$, and integrate over $d \mathbf{R} d \mathbf{V}$. Hence:

$$
\begin{array}{r}
\dot{\lambda} \frac{\left\langle\kappa(\lambda \mathbf{R}) R_{i}^{2}\right\rangle_{f_{0}}}{\left\langle R_{i}^{2}\right\rangle_{f_{0}}}-\frac{\left\langle\left[\lambda^{1-k} \kappa(\mathbf{R})-\kappa(\lambda \mathbf{R})\right] R_{i} V_{i}\right\rangle_{f_{0}}}{\lambda\left\langle R_{i}^{2}\right\rangle_{f_{0}}} \\
+\ddot{\lambda}+\lambda \omega_{0}^{2}-\frac{p}{\lambda^{3}} \omega_{0}^{2}+(p-1) \frac{1}{\lambda^{k}} \omega_{0}^{2}=0,
\end{array}
$$


where we have already introduced the parameter $p$; thus, we assume here that $\left\langle V_{i}^{2}\right\rangle_{f_{0}} /\left\langle R_{i}^{2}\right\rangle_{f_{0}}$ does not depend on $i$. To deal with the new terms we assume that $f_{0}$ presents a symmetry of the type $(\mathbb{Z} / 2 \mathbb{Z})^{d}$ for velocity variables. In a two dimensional problem this corresponds to: $f_{0}\left(r_{x}, v_{x}, r_{y}, v_{y}\right)=f_{0}\left(r_{x},\left|v_{x}\right|, r_{y},\left|v_{y}\right|\right)$. Under this hypothesis the second term in Eq. (43) vanishes. The same cancellation also happens for instance if $f_{0}$ has a spherical symmetry for space variables and the friction $\kappa(\mathbf{r})$ depends only on $|\mathbf{r}|$. These conditions are satisfied for problems with spherical symmetry. The constraint equation then reduces to:

$$
\ddot{\lambda}+\lambda \omega_{0}^{2}-\frac{p}{\lambda^{3}} \omega_{0}^{2}+(p-1) \frac{1}{\lambda^{k}} \omega_{0}^{2}+\dot{\lambda} \frac{\left\langle\kappa(\lambda \mathbf{R}) R_{i}^{2}\right\rangle_{f_{0}}}{\left\langle R_{i}^{2}\right\rangle_{f_{0}}}=0 .
$$

Note that any explicit dependence on the diffusion disappears, as its effects are averaged out. The diffusion plays a role of course in determining the stationary solution $f_{0}$, which in turns appears in the equation. The new qualitative property of Eq. (44) is an effective $\lambda$-dependent friction.

A nice feature of Eq. (40) is that the stationary solution $f_{0}$ enters in the equation only through the parameter $p$. The situation is somewhat less favorable for a spacedependent friction, since $f_{0}$ also enters into the average $\left\langle\kappa(\lambda \mathbf{R}) R_{i}^{2}\right\rangle_{f_{0}}$. However a numerical knowledge of $f_{0}$ is sufficient to use Eq. (44) in a given problem.

Eq. (44) must now be tested against direct numerical simulations.

4.2. Numerical tests. We now test the validity of Eq. (44) on academic examples of variable frictions.

One problem is the determination of the stationary profile $f_{0}$; this is a relatively easy task when friction and diffusion are constant, as $f_{0}$ is given by (3). In the present case, we have no analytical expression for $f_{0}$, and some numerical help is needed. We will actually in the following postulate a given density profile, and check that it is consistent with the numerics.

We choose to study a one dimensional plasma with $p \ll 1$, with a constant diffusion and a space-dependent friction. For a constant friction and diffusion, the density profile is then a step function; this is also a one dimensional analog of an atomic cloud in a Magneto-Optical Trap [25]. We have checked numerically that a variable friction does not change the step profile, to our numerical precision. We will then use this step profile, with cut-off length $L_{i}$ to estimate the averages in Eq. (44).

As a first test let us use the following expression for the friction:

$$
\kappa(\mathbf{r})=\left\{\begin{array}{cl}
\kappa_{0}\left(1-\frac{|\mathbf{r}|}{L_{\kappa}}\right) & \text { if }|\mathbf{r}| \leq L_{\kappa} \\
0 & \text { if }|\mathbf{r}|>L_{\kappa}
\end{array}\right.
$$

where $L_{\kappa}$ is the cut off for the friction; we do not consider negative friction here. In addition, for $|\mathbf{r}|$ larger than $L_{\kappa}$, the particles feel a diffusion without friction so that the local temperature of the system becomes infinite $(T \sim D / \kappa)$. To avoid this non-physical situation we make sure that particles stay where the friction is non zero for all time. This condition can be written as $\lambda(t) L_{i}<L_{\kappa}$. The constraint equation becomes

$$
\ddot{\lambda}+\lambda \omega_{0}^{2}-\frac{p}{\lambda^{3}} \omega_{0}^{2}+(p-1) \frac{1}{\lambda^{k}} \omega_{0}^{2}+\dot{\lambda} \kappa_{0}\left(1-\frac{3}{4} \frac{\lambda L_{i}}{L_{\kappa}}\right)=0 .
$$


Let us stress that $p \ll 1$ and we limit the discussion to systems with $\max _{|\mathbf{r}| \in \mathbb{R}}(\kappa(\mathbf{r}))=$ $\kappa_{0} \ll \omega_{0}$. Because satisfying these two conditions with constant friction yields pretty good prediction, it is reasonable to expect that it will be the same in this case.

Figs 7(a) and 7(b) compare numerical simulations with Eq. (46). In these two examples, we are in the a priori favorable situation where both $p \ll 1$ and $\max _{|\mathbf{r}| \in \mathbb{R}}(\kappa(\mathbf{r}))=\kappa_{0} \ll \omega_{0}$. We see on fig. 7(a) that the oscillation amplitude and frequency are quite well predicted; for a large amplitude oscillation, the agreement degrades after a few oscillations (see fig $7(\mathrm{~b})$ ). On this figure, the asymptotic value of $\left\langle R^{2}\right\rangle_{f} /\left\langle R^{2}\right\rangle_{f_{0}}$ seems to be different from 1 , indicating a relaxation to a stationary state different from the initial one. However, after a sufficiently long time, the system actually relaxes to $f_{0}$.

We consider now another example of variable friction:

$$
\kappa(\mathbf{r})=\kappa_{0}\left[1+\cos \left(\pi \frac{|\mathbf{r}|}{L_{\kappa}}\right)\right],
$$

where $L_{\kappa}$ represents now the first minimum of $\kappa(\mathbf{r})$. Equation (44) becomes:

$$
\begin{aligned}
& \ddot{\lambda}+\kappa_{0} \dot{\lambda}+\lambda \omega_{0}^{2}-\frac{p}{\lambda^{3}} \omega_{0}^{2}+(p-1) \frac{1}{\lambda^{k}} \omega_{0}^{2} \\
& +\frac{3}{2} \frac{\dot{\lambda} \kappa_{0}}{L_{i}^{3}} \int_{0}^{L_{i}} \cos \left(\frac{\pi \lambda R}{L_{\kappa}}\right) R^{2} d R=0 .
\end{aligned}
$$

The comparison between (48) and simulations yields similar qualitative results: the frequency is always obtained with very good precision (see Figs.7(c), 7(d) and 7(e)) at least for several oscillations. In Fig. $7(\mathrm{f})$, the condition $\kappa_{0} \ll \omega_{0}$ is relaxed, and the agreement remains almost perfect.

The main novelty of Eq. (44) with respect to Eq. (40) is the appearance of an effective non linear friction, which depends on $\lambda$. We have compared the numerical results (for $d=1, k=0$ ) with both Eq. (44) and Eq. (40), using two ad hoc effective frictions, independent of $\lambda: \kappa_{\text {eff }}^{(1)}=\langle\kappa(\mathbf{r})\rangle_{f_{0}}$ and $\kappa_{\text {eff }}^{(2)}=\left\langle\kappa(\mathbf{r}) r_{1}^{2}\right\rangle_{f_{0}} /\left\langle r_{1}^{2}\right\rangle_{f_{0}}$. The difference induced by the nonlinear friction is small, but the prediction of Eq. (44) is better, see the inset of Fig. 7(f).

\section{Conclusion}

Starting from the first equation of the BBGKY hierarchy and a scaling ansatz for the dynamics, a non-linear equation describing the breathing oscillations of trapped particles interacting via homogeneous forces was derived in [1]. The derivation and equation should be valid independently of the temperature, interaction strength, interaction range and dimensionality of the physical space, in the underdamped limit.

In this paper, we show that this equation also exactly describe the breathing dynamics of particles interacting through repulsive interactions in the zero temperature limit, for all values of the friction. We then have compared the predictions of this equation with direct numerical simulations, testing a wide range of parameters, to investigate its domain of validity. It appears that in the underdamped regime the main limitation is due to violent relaxation phenomena (sometimes called phase mixing) for long range interacting systems and two-body collisions in short interacting ones, specially for weak repulsive and attractive interactions, where they introduce damping and loss of coherence, unaccounted for in the scaling ansatz. In 
the overdamped regime, the equation is valid only at very small temperature, for repulsive interactions.

Motivated by the physics of Magneto-Optical Traps, we have extended the breathing mode theory to the case of space-dependent friction and diffusion. The predictions are again in good agreement with molecular dynamics simulations, in the underdamped and small temperature (in the repulsive case) limits.

\section{ACKNOWLEDGEMENT}

This work is supported by the ANR-09-JCJC-009401 INTERLOP project.

\section{REFERENCES}

[1] A. Olivetti, J. Barré, B. Marcos, F. Bouchet and R. Kaiser Phys. Rev. Lett. 103, 224301 (2009).

[2] D. Guéry-Odelin Phys. Rev. A 66, 033613 (2002).

[3] Y. Castin and R. Dum Phys. Rev. Lett. 76, 6 (1996).

[4] Yu. Kagan, E.L. Surkov and G.V. Shlyapnikov Phys. Rev. A 55, R18 (1997).

[5] D.H.E. Dubin Phys. Rev. Lett. 66, 2076 (1991).

[6] D.H.E. Dubin Phys. Fluids B 5, 295 (1993).

[7] Sh. Amiranashvili, M.Y. Yu and L. Stenflo Phys. Rev. E 67, 016408 (2003).

[8] D.F.V. James Appl. Phys. B 66, 181 (1998).

[9] S.A. Tatarkova, A.E. Carruthers and K. Dholakia Phys. Rev. Lett. 89, 28 (2002).

[10] V.A. Schweigert and F.M. Peeters, Phys. Rev. B 51, 7700 (1995).

[11] B. Partoens and F.M. Peeters Condens. Matter 9, 5383 (1997).

[12] S.W.S. Apolinario and F.M. Peeters, New J. Phys. 76, 115419 (2007).

[13] C. Henning, K. Fujioka, P. Ludwig, A. Piel, A. Melzer and M. Bonitz Phys. Rev. Lett. 101, 045002 (2008).

[14] T.E. Sheridan, C.R. Buckey, D.J. Cox, R.J. Merrill and W.L. Theisen Phys. Lett. A 329, 88 (2004).

[15] T.E. Sheridan Phys. Plasmas 11, 12 (2004).

[16] S. Chandrasekhar and D.D. Elbert, Monthly Notices of the Royal Astronomical Society 155, 435 (1972).

[17] The boundary between long and short range for equilibrium statistical properties is $(k-1) / d=$ 1 , which corresponds to the integrability limit of the interaction potential [19]. For dynamical properties, it seems preferable to use the present definition.

[18] G. Labeyrie, F. Michaud and R. Kaiser Phys. Rev. Lett. 96, 023003 (2006).

[19] T. Dauxois, S. Ruffo, E. Arimondo, M. Wilkens (Eds), Lecture Notes in Physics 602, Springer (2002) Dynamics and Thermodynamics of Systems with Long Range Interactions.

[20] D.H.E. Dubin and T.M. ONeil Rev. Mod. Phys. 71, 87 (1999).

[21] M.P. Allen and D.J. Tildesley Computer simulation of liquids, (Clarendon Press, Oxford, 1987).

[22] T. Buchert, Mon. Not. Roy. Astron. Soc. 254, 729 (1992).

[23] D. Lynden-Bell, Mon. Not. Roy. Astron. Soc. 136, 101 (1967).

[24] F. Bouchet and M. Corvellec, J. Stat. Mech. P08021 (2010).

[25] T. Walker, D. Sesko and C. Wieman Phys. Rev. Lett. 64, 408 (1990).

Laboratoire J. A. Dieudonné, UMR CNRS 6621, Université de Nice-Sophia Antipolis, Parc Valrose, F-06108 Nice Cedex 02, France.

Institut Non-Linéaire de Nice,UMR CNRS 6618, Université de Nice-Sophia Antipolis, France. 


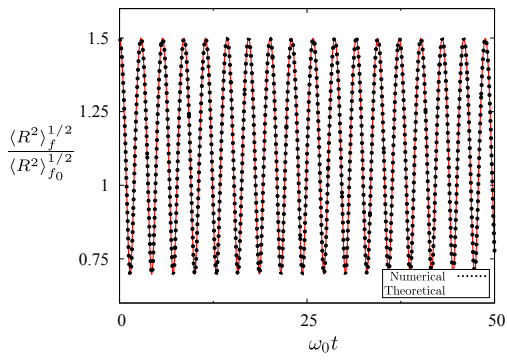

(a)

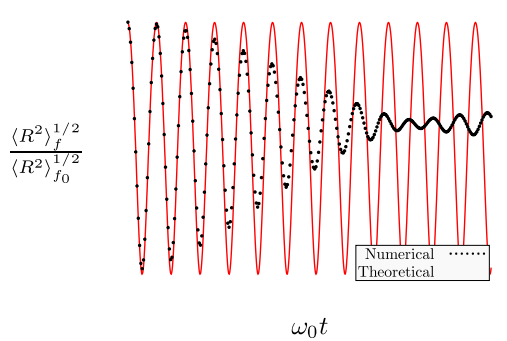

(c)

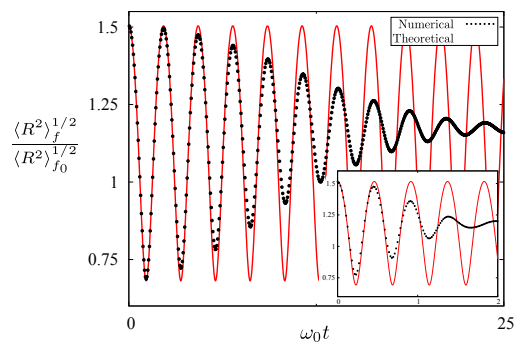

(e)

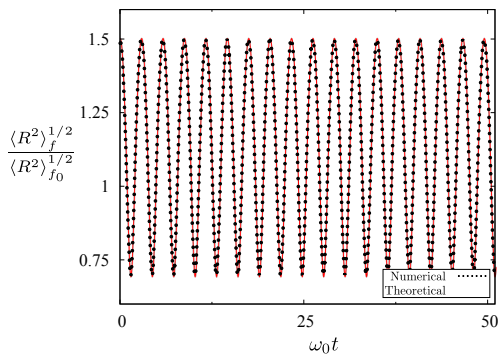

(b)

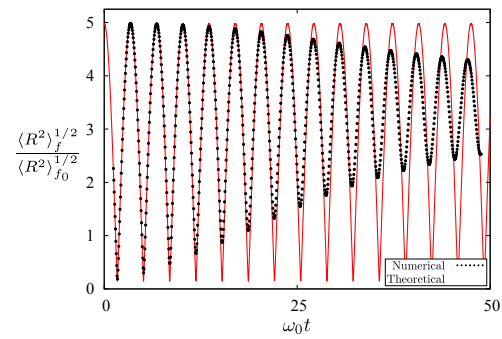

(d)

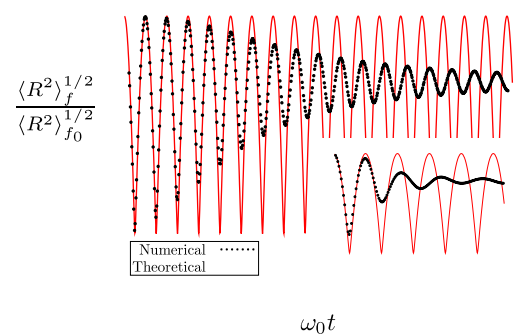

(f)

Figure 4. (color online). Microcanonical evolution of the typical size of the cloud $\left(N=4000, \omega_{0}=17.8\right)$. (a) The space dimension is $d=3$, and the interactions are repulsive. The parameters are $k=4$ (short range interaction), $p=1.2 \times 10^{-3}$ and $\left.(\lambda, \dot{\lambda})\right|_{t=0}=(1.5,0.0)$. (b) The space dimension is $d=2$, and the interactions are repulsive. The parameters are $k=4$ (short range interaction), $p=1.66 \times 10^{-1}$ and $\left.(\lambda, \dot{\lambda})\right|_{t=0}=(1.5,0.0)$. (c) The space dimension is $d=3$, and the interactions are repulsive. The parameters are $k=0$ (long range interaction), $p=4.5 \times 10^{-1}$ and $\left.(\lambda, \dot{\lambda})\right|_{t=0}=(1.5,0.0)$. (d) The space dimension is $d=3$, and the interactions are repulsive. The parameters are $k=0$ (long range interaction), $p=4.4 \times 10^{-1}$ and $\left.(\lambda, \dot{\lambda})\right|_{t=0}=(5.0,0.0)$. (e) The space dimension is $d=3$, and the interactions are attractive. The parameters are $k=0$ (long range interaction), $p=2.2$ and $\left.(\lambda, \dot{\lambda})\right|_{t=0}=(1.5,0.0)$. Same parameters for the inset except $p=70$. (f) The space dimension is $d=3$, and the interactions are attractive. The parameters are $k=0$ (long range interaction), $p=2.2$ and $\left.(\lambda, \dot{\lambda})\right|_{t=0}=(5.0,0.0)$. Same parameters for the inset except $p=70$. 
BREATHING DYNAMICS FOR SYSTEMS OF INTERACTING PARTICLES IN THE MICROCANONICAL AND CANONICAL DES

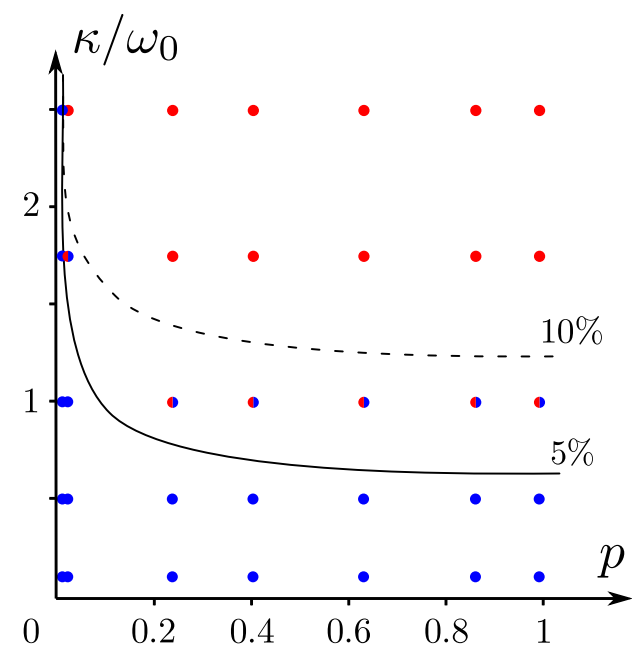

Figure 5. (color online). Validity of the scaling ansatz in the plane $\left(\kappa / \omega_{0}, p\right)$ plane. The simulations are done with $N=5000$ particles and a one-dimensional Coulombian interaction $(k=0)$ with $\omega_{0}=17.8$ and $\left.(\lambda, \dot{\lambda})\right|_{t=0}=(0.3,0.0)$. Note that the picture does not appreciably change is we consider instead $N=1000$ or $d=2$, 3. Blue circle: frequency error $\leq 5 \%$; half-blue/red circle: $5 \%<$ error $\leq 10 \%$; red circle: error $>10 \%$; solid and dashed line represent respectively the qualitative boundaries for $5 \%$ and $10 \%$ frequency error. For a definition of the frequency error, see text. 


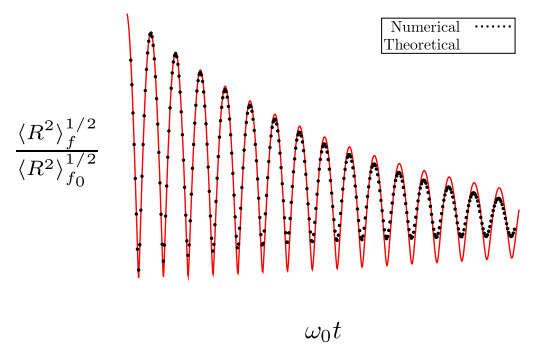

(a)

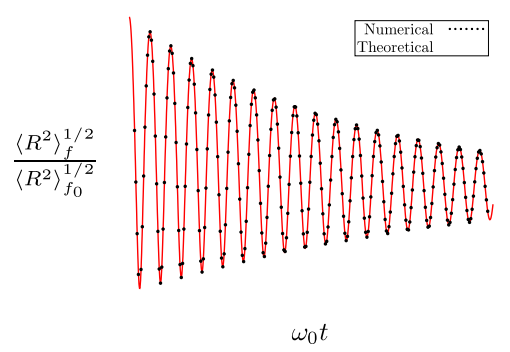

(c)

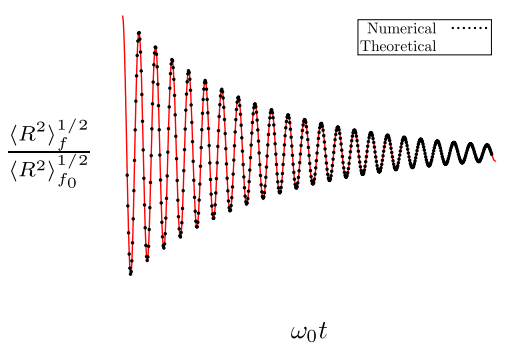

(b)

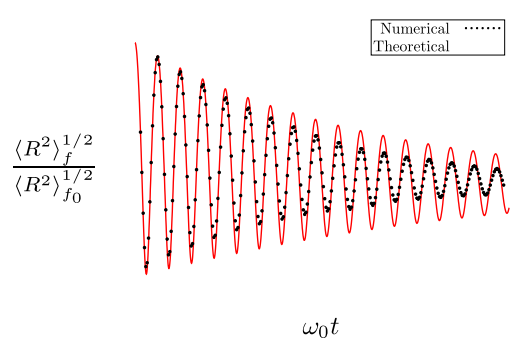

(d)

Figure 6. (color online). Evolution of the typical size of the cloud in the underdamped case $\left(N=4000, \kappa / \omega_{0}=5.6 \times 10^{-2}\right)$. (a) The space dimension is $d=3$, and the interactions are repulsive. The parameters are $k=1$ (long range interaction), $p=5.0 \times 10^{-1}$ and $\left.(\lambda, \dot{\lambda})\right|_{t=0}=(5.0,0.0)$. (b) The space dimension is $d=3$, and the interactions are repulsive. The parameters are $k=1$ (long range interaction), $p=1.5 \times 10^{-3}$ and $\left.(\lambda, \dot{\lambda})\right|_{t=0}=(1.2,0.0)$. (c) The space dimension is $d=3$, and the interactions are repulsive. The parameters are $k=4$ (short range interaction), $p=1.2 \times 10^{-3}$ and $\left.(\lambda, \dot{\lambda})\right|_{t=0}=(1.5,0.0)$. (d) The space dimension is $d=2$, and the interactions are repulsive. The parameters are $k=4$ (short range interaction), $p=6.3 \times 10^{-1}$ and $\left.(\lambda, \dot{\lambda})\right|_{t=0}=(1.5,0.0)$. 
BREATHING DYNAMICS FOR SYSTEMS OF INTERACTING PARTICLES IN THE MICROCANONICAL AND CANONICAL DES

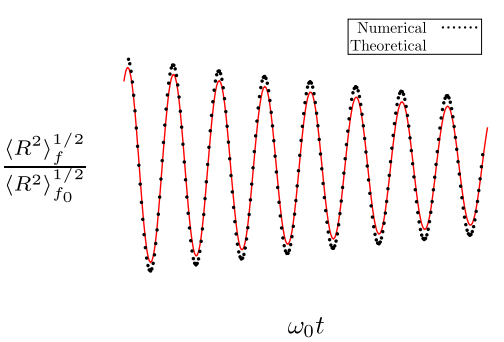

(a)

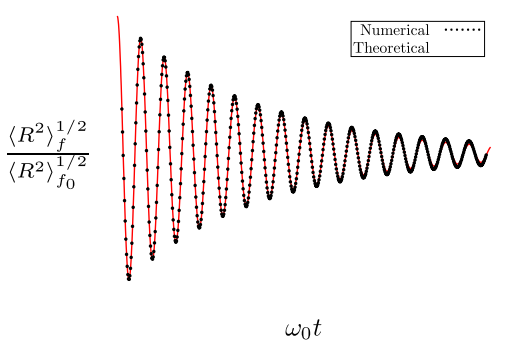

(c)

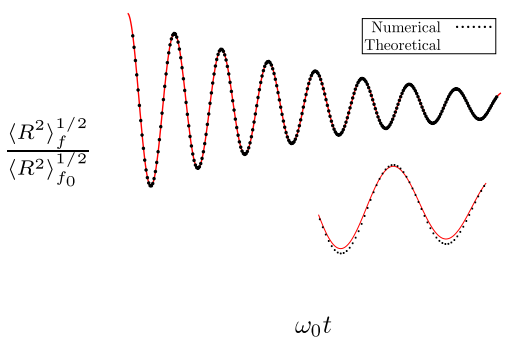

(e)

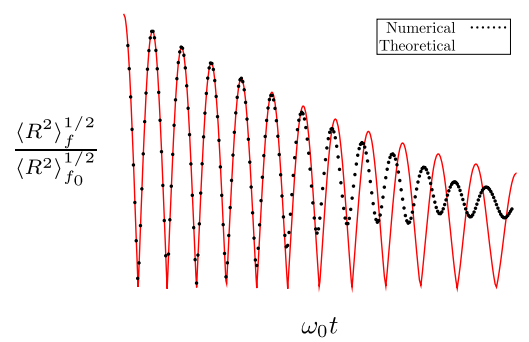

(b)

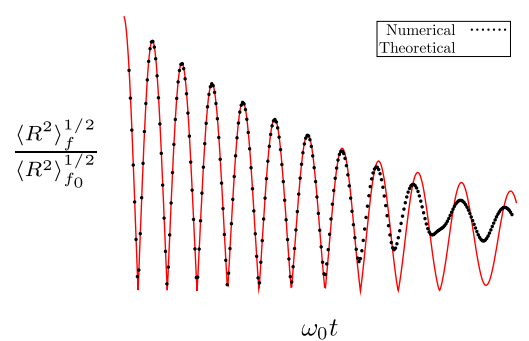

(d)

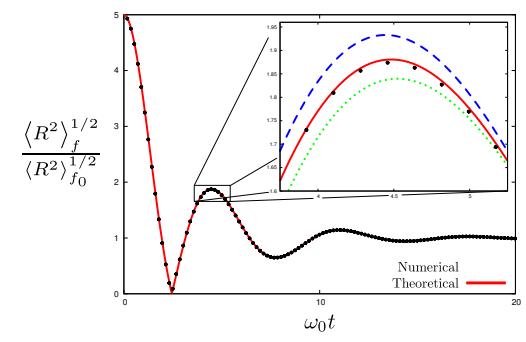

(f)

Figure 7. (color online). Evolution of the typical size of the cloud with non homogeneous friction in a one dimensional Coulombian system $\left(N=4000, d=1, k=0, L_{i}=12.5\right.$ and $\left.p=6 \times 10^{-5}\right)$. (a) The friction profile is given by (45). Parameters are $\kappa_{0} / \omega_{0}=$ $5.6 \times 10^{-2}, L_{\kappa}=1.25 \times L_{i}$ and $\left.(\lambda, \dot{\lambda})\right|_{t=0}=(1.2,0.0)$. (b) The friction profile is given by (45). Parameters are $\kappa_{0} / \omega_{0}=5.6 \times 10^{-2}$, $L_{\kappa}=5.0 \times L_{i}$ and $\left.(\lambda, \dot{\lambda})\right|_{t=0}=(5.0,0.0)$. (c) The friction profile is given by (47). Parameters are $\kappa_{0} / \omega_{0}=5.6 \times 10^{-2}, L_{\kappa}=0.25 \times L_{i}$ and $\left.(\lambda, \dot{\lambda})\right|_{t=0}=(1.2,0.0)$. (d) The friction profile is given by $(47)$. Parameters are $\kappa_{0} / \omega_{0}=5.6 \times 10^{-2}, L_{\kappa}=4.0 \times L_{i}$ and $\left.(\lambda, \dot{\lambda})\right|_{t=0}=$ $(5.0,0.0)$. (e) The friction profile is given by $(47)$. Parameters are $\kappa_{0} / \omega_{0}=5.6 \times 10^{-2}, L_{\kappa}=0.25 \times L_{i}$ and $\left.(\lambda, \dot{\lambda})\right|_{t=0}=(1.2,0.0)$. (f) The friction profile is given by (47). The dashed blue curve and dotted green curve in the inset are respectively obtained from (44) substituting $\kappa(\lambda \mathbf{R})$ by $\langle\kappa(\mathbf{R})\rangle_{f_{0}}$ and $\kappa(\mathbf{R})$. Parameters are $\kappa_{0} / \omega_{0}=5.6 \times 10^{-1}, L_{\kappa}=0.25 \times L_{i}$ and $\left.(\lambda, \dot{\lambda})\right|_{t=0}=(5.0,0.0)$. 